\title{
Finite Element Based Surface Roughness Study for Ohmic Contact of Microswitches
}

\author{
Hong Liu, Dimitri Leray \\ Institut Clément Ader, INSA \\ Univ de Toulouse \\ $\&$ \\ CNRS, LAAS \\ Univ de Toulouse, INSA, LAAS \\ Toulouse, France \\ E-mail : hliu@laas.fr \\ Patrick Pons \\ CNRS, LAAS \\ Univ de Toulouse, LAAS \\ Toulouse, France
}

\author{
Stéphane Colin \\ Institut Clément Ader, INSA \\ Univ de Toulouse \\ Toulouse, France \\ Adrien Broué \\ NOVAMEMS, c/o CNES \\ \& \\ CNRS, LAAS \\ Univ de Toulouse, LAAS \\ Toulouse, France
}

\begin{abstract}
Finite element method (FEM) is used to model ohmic contact in microswitches. A determinist approach is adopted, including atomic force microscope (AFM) scanning real contact surfaces and generating rough surfaces with three-dimensional mesh. FE frictionless models are set up with the elastoplastic material and the simulations are performed with a loadingunloading cycle. Two material properties, gold and ruthenium, are studied in the simulations. The effect of roughness is investigated by comparing the models with several smoothing intensities and asperity heights. The comparison is quantitatively analyzed with relations of force vs. displacement, force vs. contact area and force vs. electrical contact resistance (ECR); further the evolution of spots in contact during a loadingunloading cycle is studied.
\end{abstract}

Keywords-ohmic contact, spherical rough surface, determinist finite element model, loading-unloading

\section{INTRODUCTION AND MOTIVATIONS}

\section{A. Contact of Rough Surfaces}

Roughness of contact surfaces is a great concern for the contact resistance of microswitches. Many researchers have devoted to analyzing and modeling the contact behavior and calculating the resistance. Several main methods are following, statistical [1-4], fractal [5-7], multi-scale [8-9] and determinist model [10]. The elastic or elastic-plastic deformation is considered in the above models, and the contact condition and material properties are studied, too [11].

However, all these works, except [10], deal with the contact of flat rough surfaces. In our microswitches [12], contact occurs between a spherical bump and a flat bridge. Since the roughness of the bridge is much inferior to that of the bump, only the roughness of the bump is taken into account; Which gives a contact between a spherical rough surface and a flat surface. Unlike contact between nominally flat surfaces, in a spherical rough contact, both contact asperities and the bulk deform under normal load [13]. Several works on spherical contact are found in the literature, while most of them deal with the contact between a rough flat and a smooth spherical [1415], further the displacement is assumed to be elastic and/or purely plastic. The work [16] studied the spherical rough contacts, with the deformation of asperities assumed to be purely plastic. More recently, references are given as, [13] and [17] analyzed the contact of a rough sphere and a rigid flat, with elastic, elastic-plastic and fully plastic regime considered for the deformation of the sphere and the asperities; however, the roughness of the sphere was transferred to the flat. Reference [18] found that the flatten models predict larger contact areas and higher loads than the indentation model at the same interference, and they cannot be replaced by each other. Our preceding simulations also proved that the flatten model is stiffer than the indentation model.

\section{B. Unloading a Contact}

Repeated loading and unloading of rough surfaces leads to residual stress and plastic strain in each contact asperity, which raises the difficulty of modeling. Many researchers have studied loading-unloading of a sphere in contact with a flat. Reference [19] developed a model for unloading of an elasticplastic loaded sphere; analytical expressions for the dimensionless contact load and contact area vs. dimensionless interference were derived by best fitting of numerical FEM results.

Experimental studies on spherical contact were carried out by [20]; the relation between real contact area and normal load during loading and unloading matched very well the theoretical results [19], whereas, roughness was not considered. Further, in [21], the effect of adhesion on the deformation is considered for material $\mathrm{Au}$ and $\mathrm{Ru}$ in the finite element model of loading and unloading.

All of the above mentioned studies assume elastic-fully plastic material, if not elastic material. In [19], only $2 \%$ elastic linear hardening was selected in the study, which is very close to the elastic-perfectly plastic case. In [23], the author 
discussed the effect of strain hardening for the unloading of a sphere against a rigid flat, and concluded that the generalized solution cannot be applicable for all kinds of materials; furthermore, all of these studies deal with contact between a sphere and a rigid flat, no roughness is considered.

In the recent work [22], the author discussed a spherical contact, considering the material from elastic to elastic-fully plastic, and gets the load-deformation equation at unloading and the residual interference after complete unloading, whereas no roughness is considered.

Reference [24] studied the unloading of an elastic-plastic contact of rough surfaces. Relations for dimensionless mean separation and real contact area vs. contact load during one loading-unloading cycle were obtained. However, as the study is based on the previous work [19], the material property is elastic-fully plastic.

\section{Literature Review and Structure of the Paper}

As can be seen from the above literatures, in terms of rough contact modeling, elastic-plastic material property is lacking for the spherical contact; while in terms of the loadingunloading cycle, no spherical rough surfaces were studied.

The present study adopts a determinist method as the easiest way to properly describe the roughness [10]. Atomic force microscopy (AFM) is used to measure the topography of a microswitch bump, which takes well the topographies of micro-scale asperities and macro-scale bump form. Using the AFM scan data, finite element (FE) contact model is created in ANSYS $^{\mathrm{TM}}$ package 11.0.

Although FE models can provide an accurate solution for the elastic-plastic contact problem, obtaining accurate results might require very long computations [25]. This study first considers several levels of mesh grid size, in order to get relatively accurate results with less time consuming. Secondly, asperity height is assumed to be the main roughness parameter; the influence of asperity height on contact is investigated.

A number of materials can be used as contact materials in ohmic microswitches. This study considers gold $(\mathrm{Au})$ and ruthenium $(\mathrm{Ru})$. This choice allows us to study the effects of material hardness. The material property is defined as elasticplastic with strain hardening. The contact stiffness, i.e. contact force vs. bulk displacement, the evolution of contact area, mean contact pressure, and also electrical contact resistance (ECR) during a loading-unloading cycle are derived from the simulations. Some dimensionless results are compared with the theoretical results from the literatures [19], [26].

This paper is organized as follows: the AFM scan and the FE model are presented in section II. The material property, boundary conditions, loading process and contact resistance calculation are presented in section III. Simulation results and discussions on contact behavior are detailed in section IV. Contact resistance and the evolution of contact spots during loading-unloading are analyzed in section V. Summary and conclusions are laid in section VI.

\section{DESCRIPTION OF CONTACT TOPOGRAPHY AND FE MODEL}

\section{A. Topography of Contact Profile in Microswitches}

A real microswitch is used for AFM scanning. Fig. 1(a) shows the profile of the device. Contact members are as follows:

- Bridge is made of gold, thickness is $4 \mu \mathrm{m}$

- Bump is $4 \mu \mathrm{m}$-wide, coated with $1 \mu \mathrm{m}$-thick gold.

Since the microswitch works under the weak force, it is the asperities in contact, also called a-spots, which are in the dimension of about hundred or even tens of nanometers [27], so the high resolution is required to properly map the surface topography.

A $4 \mu \mathrm{m}$-wide square AFM scan is carried out on the microswitch bump. Scan X-Y grid is set to 256 lines, which gives a $15.6 \mathrm{~nm}$ horizontal resolution. This horizontal resolution will be proved to be sufficient for modeling contact behavior, and also to evaluate ECR. Fig. 1 (b) shows the profile of topography, AFM data are treated by Matlab.

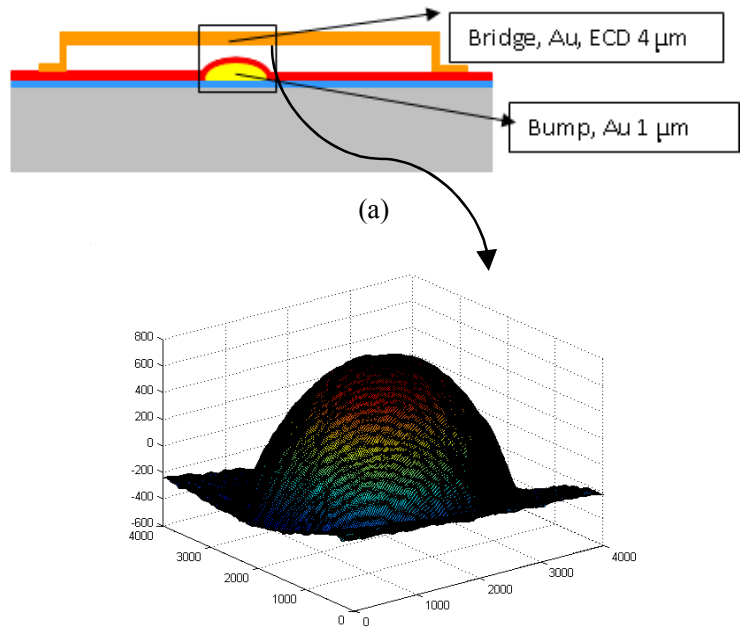

(b)

Figure 1. (a) Structure profile of the microswitch, (b) AFM scan of the microswitch bump

\section{B. FE Model for Contact}

The contact parts are modeled as deformable bodies. The topside of the bottom body is built using AFM data. It includes the shape and roughness of the microswitch bump. The bottom surface of the upper body has infinitely flat surface.

A 3-Dimensional (3D) FE model is built in ANSYS ${ }^{\mathrm{TM}}$. Both deformable bodies are modeled using 3D tetrahedral solid element SOLID187, which has plasticity, stress stiffening, large deflection and large strain capabilities. 3D surface-tosurface contact element CONTA174 and target element TARGE174 are used to mesh respectively bump and bridge contact surfaces. These elements are selected to consider the large deflection and nonlinear behavior of contact asperities. The augmented Lagrange method is used to seek contact in the 
simulations, and the large deformation is included during the calculation.

Boundary conditions include (see Fig. 2):

- Bottom surface of the lower body is clamped.

- All nodes of upper body top surface are linked so that they have the same displacement in the $Z$ direction only.

- Pressure is applied uniformly on the upper surface of the top body.

\section{1) Contact of rough surfaces}

Fig. 2(a) shows a coarse mesh of the whole bump and the corresponding part of the bridge. However, as large memory is required, the computer failed to fulfill the simulation with finest resolution of the AFM scan. Therefore, the first measure to limit the computation time is to reduce the modeled volume.

Two models are created:

- Whole bump model, with scan dimension of $4 \times 4 \mu \mathrm{m}$.

- $\quad$ Reduced model, with smaller dimension of $1.2 \times 1.2$ $\mu \mathrm{m}$, includes the top zone of the bump, which has the highest points in contact (see Fig. 2(b)).

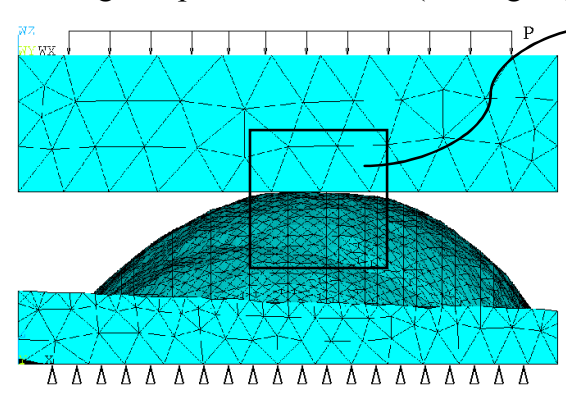

(a)

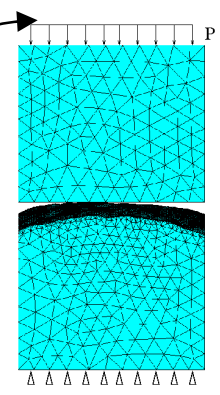

(b)
Figure 2. FE contact model with rough surface based on the AFM data for (a) the whole bump (b) the reduced zone

The preliminary simulations showed that the reduced model gives the precise results in terms of contact behavior and contact resistance. The following simulations are carried out using the reduced model, as Fig. 2(b) presents.

\section{2) Contact of smooth surfaces}

The counterpart smooth-smooth (S-S) model also consists of two deformable bodies. Here the bump is modeled as a smooth sphere cap.

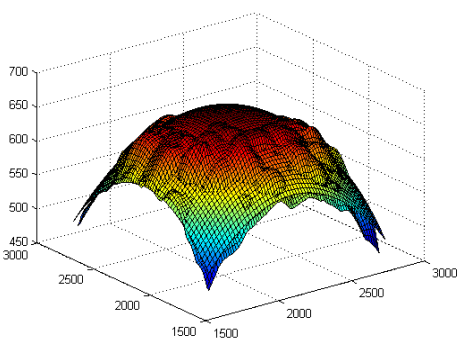

(a)

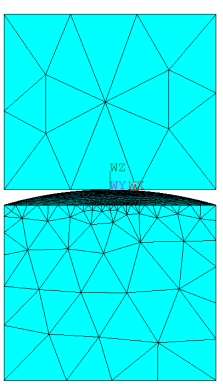

(b)
Figure 3. (a) Bump profile: AFM data \& least squares sphere, (b) S-S contact model.
Least squares fitting is used to define the sphere radius. Fitting is carried out using the data of the reduced model, i.e. $1.2 \times 1.2 \mu \mathrm{m}^{2}$, the resulting value is $\mathrm{Rs}=2.1 \times 10^{3} \mathrm{~nm}$. Fig. 3(a) shows that the sphere cap surface describes exactly the general shape of the reduced zone at the top of the bump. The $\mathrm{S}-\mathrm{S}$ model is shown on Fig. 3(b).

\section{CONTACT MECHANICS AND ECR CALCULATION}

\section{A. Material Properties and Load Protocol in FE Model}

As it is the asperities that carry the load in the microcontact, they experience plastic deformations almost as soon as the contact occurs, and, sometimes, in the fully-plastic form. Reference [28] shows the importance of the tangent module, i.e. strain hardening, for contact behavior. However, a bilinear isotropic hardening property was adopted in their simulations, which ignored the ultimate strength of material.

Two material property models are investigated in our simulations: bilinear isotropic and multilinear isotropic, respectively BISO and MISO options in ANSYS (see Fig. 4(a)). The results on contact pressure show a significant difference between those, which proves the importance to consider the ultimate strength. Material properties for the numerical models are listed in Table 1.

TABLE I. MATERIAL PROPERTIES IN THE FE MODEL

\begin{tabular}{|c|c|c|}
\hline Properties (unite) & $\mathrm{Au}$ & $\mathrm{Ru}$ \\
\hline Yong's modulus $E(\mathrm{GPa})$ & 80 & 447 \\
\hline Poisson's ratio $v$ & 0.42 & 0.3 \\
\hline Yield strength $\sigma_{y}(\mathrm{GPa})$ & 0.3 & 3.6 \\
\hline Ultimate strength $\sigma_{u}(\mathrm{GPa})$ & 0.36 & 4.4 \\
\hline Tangent modulus $E t(\mathrm{GPa})$ & 10 & 25 \\
\hline $\begin{array}{c}\text { Electrical resistivity } \\
\left(10^{-9} \Omega \times \mathrm{m}\right)\end{array}$ & 22.14 & 71 \\
\hline
\end{tabular}

A loading path such as "zero - max force - zero"(see Fig. 4 (b)) is used to study the effect of strain hardening and the residual stress. The maximum force is taken as $145 \mu \mathrm{N}$, which is the work force in the microswitchs [12].

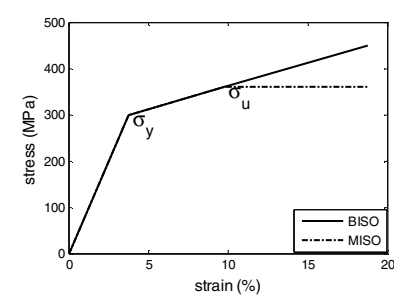

(a)

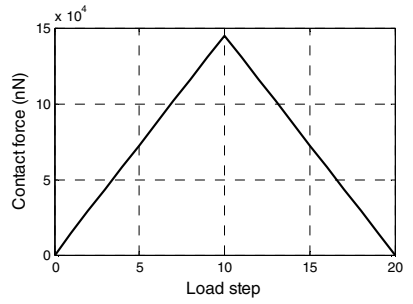

(b)
Figure 4. (a) BISO/MISO material property options in ANSYS, (b) a loadunload cycle in the simulations.

\section{B. Contact Resistance Calculation}

Contact occurs at a finite number of spots, which results in the constriction resistance. FE contact results are exported as text files, then Matlab is used to gather the number of spots and the area of each contact spot, and finally the constriction resistance is calculated. 
In the case of a unique contact spot, the ECR is calculated according to its radius $a$. Comparing the contact radius to the electron mean free path, the following equations (1-4) allow us to calculate the constriction resistance in three electrical transport regimes: diffusive, ballistic and intermediary, for a single spot:

$$
\begin{aligned}
& R_{D}=\rho / 2 a \\
& R_{B}=4 \rho \lambda / 3 \pi a^{2} \\
& R_{\text {int }}=f\left(\frac{\lambda}{a}\right) R_{D}+R_{B} \\
& f\left(\frac{\lambda}{a}\right)=\frac{1+0.83(\lambda / a)}{1+1.33(\lambda / a)}
\end{aligned}
$$

Where is the electrical resistivity and is the electron mean free path. $R_{D}$ and $R_{B}$ represent the diffusive and ballistic transport regimes respectively, and $R_{i n t}$ for the intermediary regime. $f(/ a)$ is an interpolation function, which accounts for the transition between the two resistance regimes.

When multiple spots are in contact, the effective contact resistance depends on the radii of the spots and their distribution. Even there are some formulae to calculate the ECR for this case [1] [29], it is difficult to cooperate with all of the spots in different transport regimes. In the study, as [30] proposed, a lower and upper limit is defined for ECR.

For the lower limit, it is assumed that the contact spots are in parallel, and do not interact with each other; for the upper limit, the ECR is obtained by replacing all contact asperities with one single spot while keeping the contact area constant, and the effective radius $a_{\text {eff }}$ is used. The ECR limits can be calculated with (5-6).

$$
\begin{aligned}
& \frac{1}{R_{l}}=\sum_{i=1}^{N} \frac{1}{R_{c i}} \\
& R_{u}=f \frac{\rho_{a v}}{2 a_{e f f}}+\frac{4 \rho_{a v} \lambda}{3 \pi a_{e f f}^{2}}
\end{aligned}
$$

Where $N$ is the number of asperity contact and ${ }_{a v}$ is an average resistivity. $R_{c i}$ is the resistance of contact spot $i$, and the subscripts $l$ and $u$ represent the lower and upper limits for the contact resistance.

\section{Contact Mechanics Analysis}

This section presents the results on contact mechanics, i.e. contact force vs. displacement, evolution of contact area and mean contact pressure for a loading-unloading cycle. To study the influence of the roughness on contact, two parameters are investigated: mesh grid size and asperity height.

Simulations are carried out with two material parameters: gold and ruthenium. These two materials are used for ohmic contact in microswitches and offer largely different hardness.

\section{A. Contact Mechanics for Gold}

\section{1) Mesh resolution}

Mesh resolution is very important for the precision of the numerical simulations. However, it requires much more time for the finer meshing, and sometimes, it gives very similar results while the mesh is orders of magnitude finer[26], [31].

In this section, the sensitivity of mesh resolution is investigated. It must be noted that a bigger mesh grid size smoothens the rough contact surface. Generally speaking, it must be kept in mind that the less smoothing, the more distinct asperities in contact, and the higher the asperities.

The reduced model with finest mesh leads to:

- 5929 nodes on the contact surface

- $\quad$ More than 500000 elements

- Over 100 hours solving time

Therefore, a series of models with coarser meshes are built, and also the S-S contact. Grid sizes for contact segment are:

\section{$15.6 \mathrm{~nm}$, model named F1}

\section{$32 \mathrm{~nm}$, named F2}

$96 \mathrm{~nm}$, named F6

S-S model, with $15.6 \mathrm{~nm}$ grid size

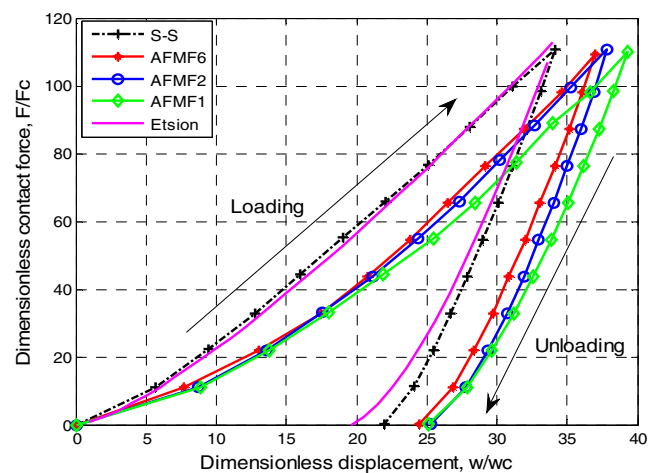

(a)

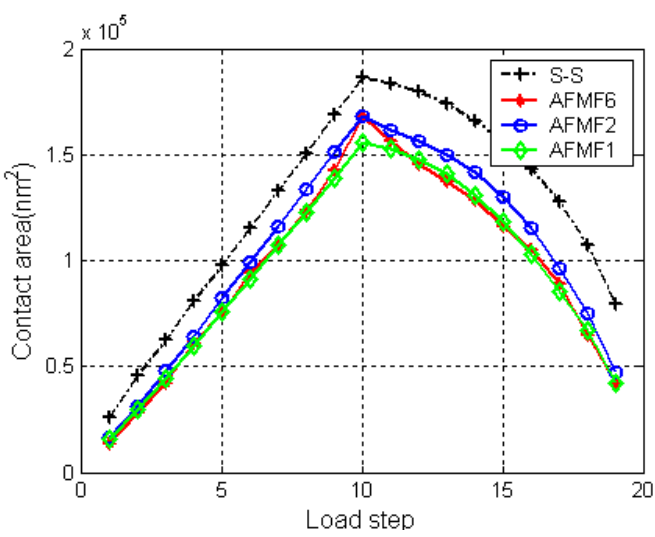

(b) 


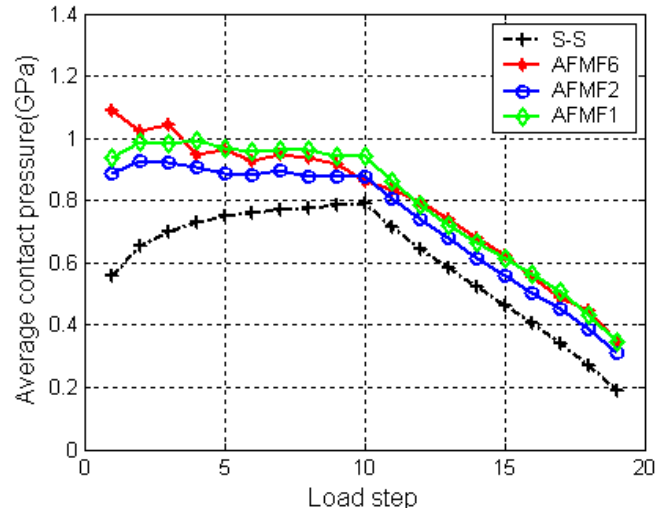

(c)

Figure 5. Simulation results for Au-Au contact with varying mesh resolution: $15.6 \mathrm{~nm}, 32 \mathrm{~nm}, 96 \mathrm{~nm}$, and S-S contact, in terms of (a), dimensionless force vs. dimensionless displacement, (b) total contact area vs. load step index, (c) mean contact pressure vs. load step index

The results for Au are presented in Fig. 5. The results for SS contact matches well the theoretical results by [19]. This confirms the correctness of both the loading-unloading formula in [19] and our numerical model.

With the formula of $[31,19]$ the critical interference $\omega_{c}$ is $0.574 \mathrm{~nm}$ for the S-S model, and the contact begins the elasticplastic deformation at the interference of $6 \omega_{c}$, i.e. $3.5 \mathrm{~nm}$. Plastic strain occurs heavily for all the models. After unloading, permanent deformation is at least $12 \mathrm{~nm}$, for about $20 \mathrm{~nm}$ of the maximum displacement.

Contact tangent stiffness, as defined by the slope of force vs. displacement, is found to be greater during unloading than loading. This is a consequence of residual deformation due to plastic strain. While comparing different models, much smaller differences are found during unloading than that of loading. This can be explained by the fact that the area of the plastically flattened contact zone depends little on the initial surface profile. This fact is to be related to the deformation being heavily plastic during loading.

The contact area is almost proportional with contact force during loading and nonlinear during unloading. This is logical seeing that the contact pressure reaches the material hardness very quickly after the beginning of loading, so the contact area can be calculated by $A=F / H$ approximately. On the contrary, the contact pressure decreases gradually during unloading. The contact pressure is hence smaller during unloading, and the contact area is larger.

Comparing with the rough models and the smooth model, one could expect that the rough surface means greater deformation and smaller contact area; hence, higher contact resistance may be obtained.

As Fig. 5 suggests, resolution of model F2 is used for the next section: results are very close to those with $F 1$, although there are four times fewer nodes on contact surface, which yields much less computing times.

\section{2) Asperity height}

Second roughness parameter in the study is the asperity height. All $\mathrm{Z}$ coordinates of AFM scan data are multiplied by a parameter factor, taken as 1,2 or 4 for our tests, and the name of the respective model is $\mathrm{U} 1, \mathrm{U} 2$ and $\mathrm{U} 4$.

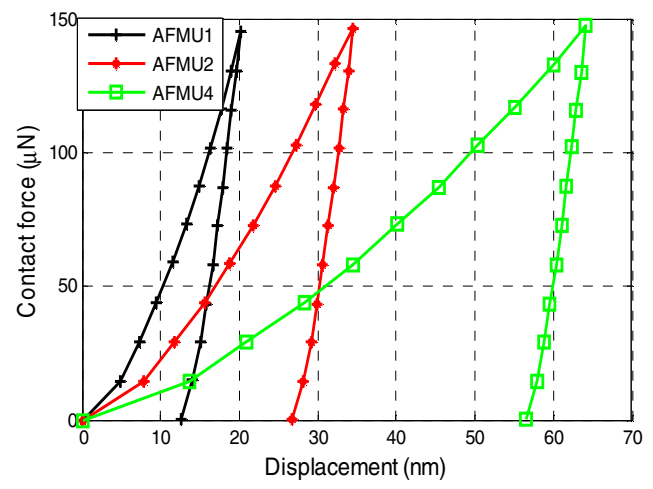

(a)

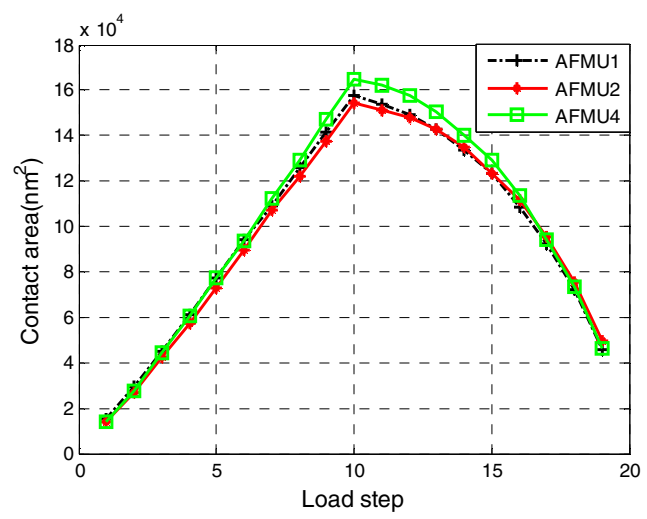

(b)

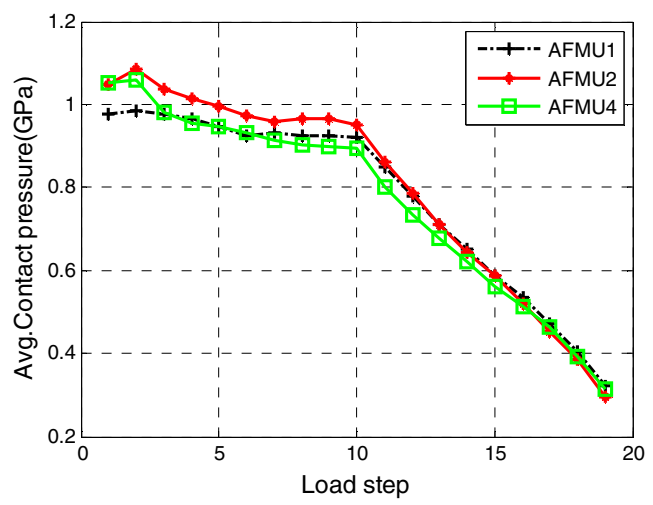

(c)

Figure 6. Simulation results for Au-Au contact with varying asperity height coefficient, (a) force vs. displacement, (b) total contact area vs. load step index, (c) mean contact pressure vs. load step index

Simulations are carried out with $32 \mathrm{~nm}$ mesh grid size (F2). The results are shown in Fig. 6. Significant difference is found for contact stiffness during loading, which is feebler with higher asperities, while they are close to each other during unloading, hence the difference between loading and unloading is more profound with higher asperity height. 
Although a significant difference is found for the stiffness, the difference for the contact area is very small, considering that the asperities have reached their hardness (Fig. 6 (c)).

\section{B. Contact Mechanics for Ruthenium}

Ohmic microswitches often use $\mathrm{Ru} / \mathrm{Ru}$ or $\mathrm{Ru} / \mathrm{Au}$ contact to provide better electrical resistance stability, in spite of a higher bulk resistivity. The same set of simulations is carried out using $\mathrm{Ru}$ mechanical properties as material input.

\section{1) Mesh resolution}

Fig. 7 presents the effect of mesh resolution. Compared with the theoretical results, significant deviation with the results by [19] is found, while the plot matches very well with the results of [26]. This indicates that [19] does not fit well for small interference case; indeed, the authors wrote that the error involved in the prediction can reach $18 \%$ at the low force.

According to [26], elastic deformation prevails up to 5.5 $\mathrm{nm}$ interference, i.e. dimensionless interference $\omega / \omega_{c}=1.9$. The smooth model undergoes hardly any plastic deformation. Conversely, because of the existence of asperities, the rough models experience plasticity. Since ruthenium is harder than gold, much less residual deformation is found after unloading. That explains why Fig 7(b) and 7(c) show almost symmetrical behavior during loading and unloading.

Fig. 7(c) shows that contact pressure increases gradually during loading; the hardness has not reached immediately, even with rough models, contrary to what gold simulation results show.

While comparing the S-S model and the rough contact models, more differences are found for contact area and contact pressure than the gold case.

Generally speaking, ruthenium models show that mesh resolution has a higher impact on contact pressure. This suggests that the accurate results for $\mathrm{Ru}$ require finer mesh grid than for $\mathrm{Au}$. Unfortunately, solving the models with finest mesh (F1) failed. F2 model is used in the subsequent analysis.

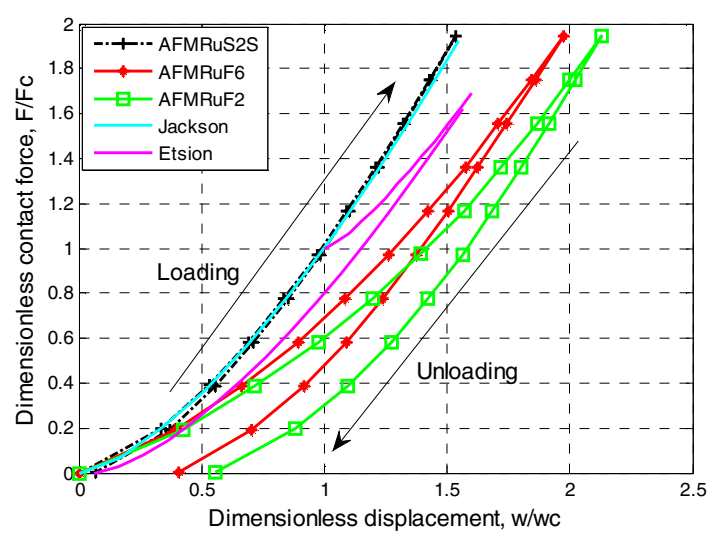

(a)

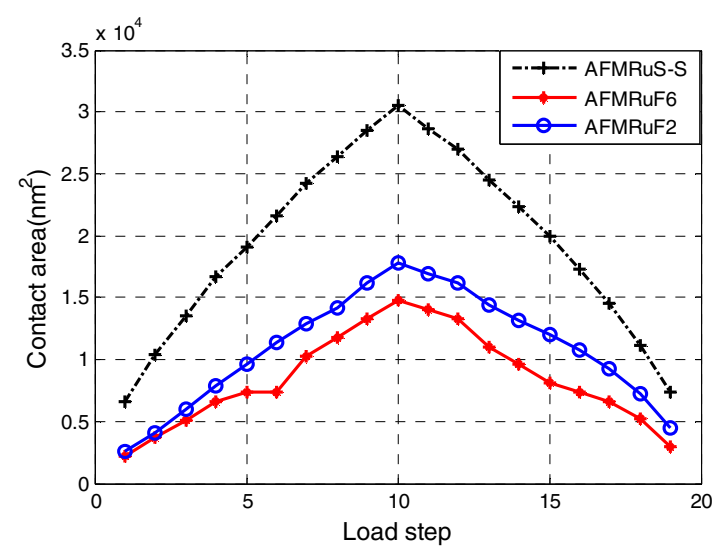

(b)

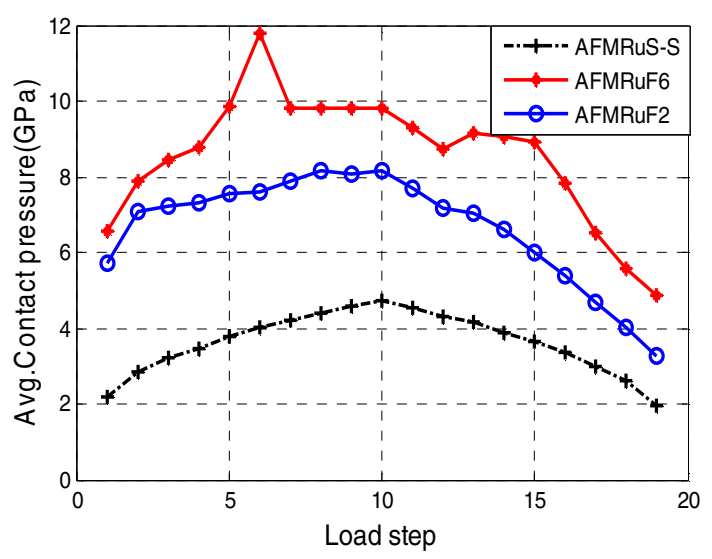

(c)

Figure 7. Simulation results for Ru-Ru contact with varying mesh resolution $32 \mathrm{~nm}, 96 \mathrm{~nm}$, and smooth-smooth contact, in terms of (a), dimensionless force vs. dimensionless displacement, (b) total contact area vs. load step index, (c) mean contact pressure vs. load step index

\section{2) Asperity height}

Fig. 8 presents the results for varying asperity height coefficient. Like the results for $\mathrm{Au}$, the contact stiffness is very different during loading, while close during unloading.

Compared to the $\mathrm{Au}-\mathrm{Au}$ contact results, less residual deformation is found, and the difference on contact stiffness between loading and unloading is smaller.

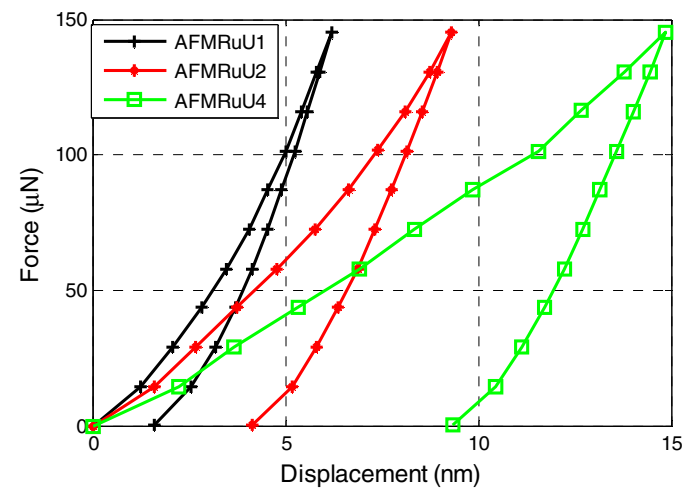

(a) 


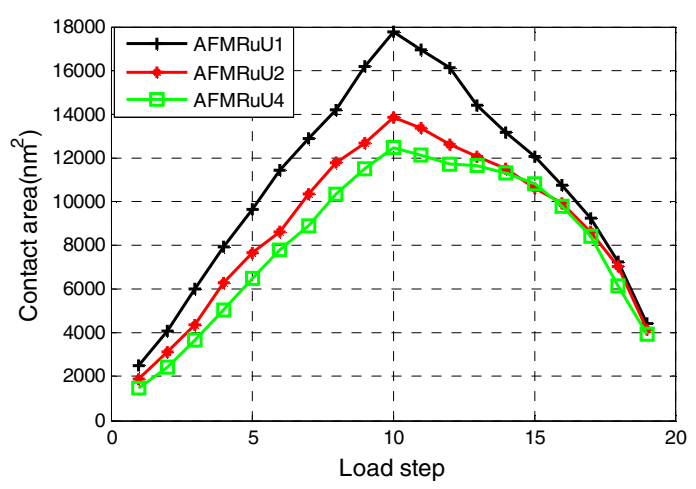

(b)

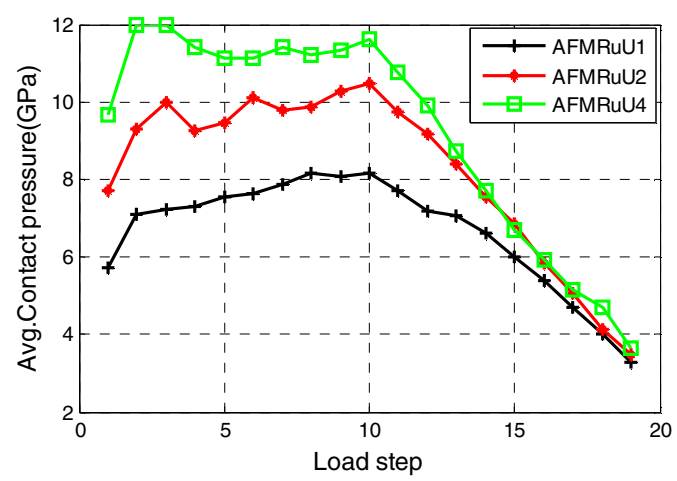

(c)

Figure 8. Simulation results for Ru-Ru contact with varying asperity height coefficient (a), force vs. interference, (b) total contact area vs. load step index, (c) mean contact pressure vs. load step index

As higher asperity height means larger value of plasticity index $\psi$ [24], it makes the contact more plastic, so they could support more contact pressure, and less contact area is required.

Comparing the results of different asperity heights, the differences on contact pressure and contact area are more significant than for $\mathrm{Au}$.

\section{CONTACt Resistance AND EVOLUtion OF CONTACT SPOTS}

\section{A. Influence of Roughness on Contact Resistance}

Contact resistance is a current issue for microswitches, and predicting resistance accurately is of great interest. The influence of mesh resolution on constriction resistance computation is the focus of this section.

As explained in part III, the upper and lower limits are calculated for the contact resistance. Fig. 9 (a) and 9 (b) show the evolution of ECR with contact force for $\mathrm{Au}-\mathrm{Au}$ contact, with the coarsest and the finest mesh. The boundary becomes looser as the meshing becomes finer. A simple explanation is brought by the number of contact spots, see Fig. 11. Indeed, the coarser the mesh, the less the number of contact spots, and the closer the ECR limits. This matches the conclusion in [31].

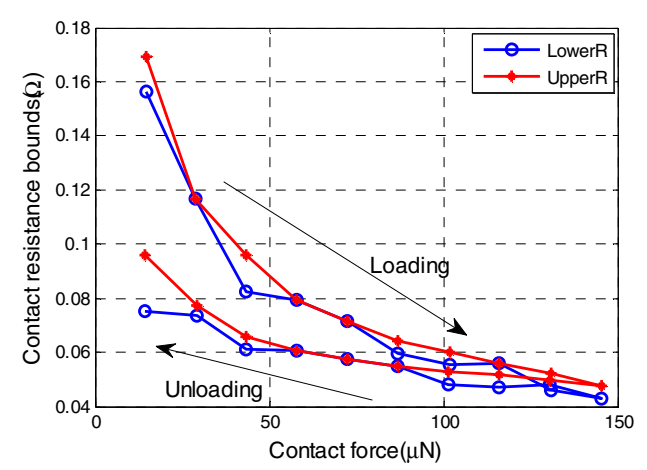

(a)

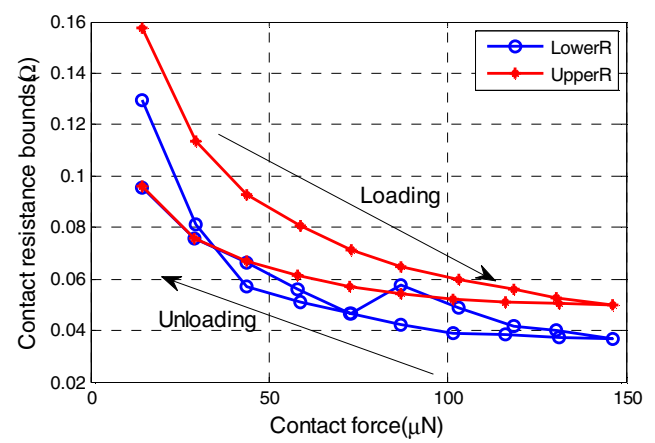

(b)

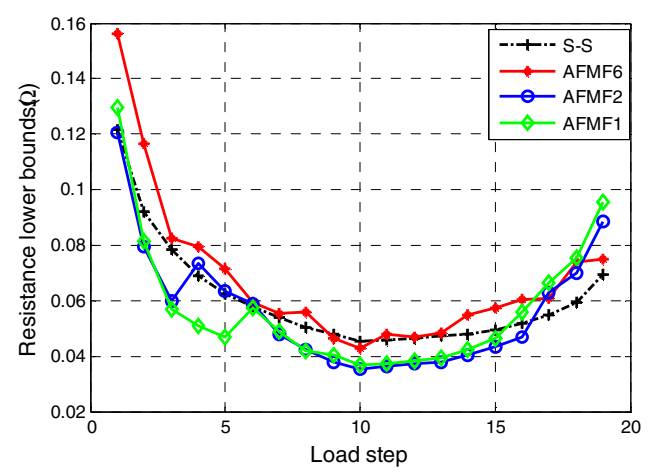

(c)

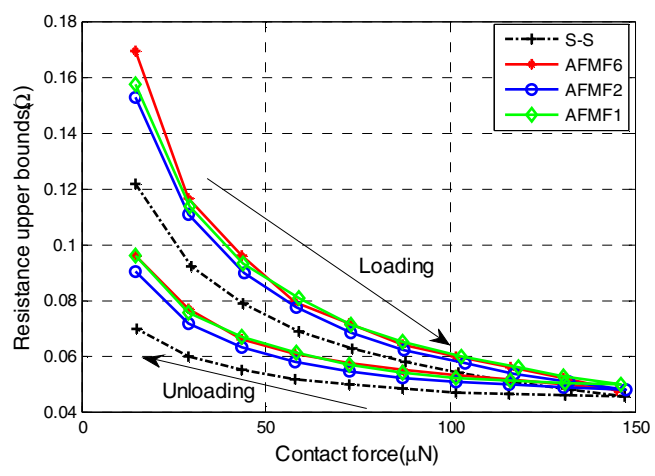

(d)

Figure 9. Contact resistance for Au-Au contact: ECR limits vs. force for the models with the (a) coarsest mesh and (b) finest mesh; ECR boundary vs. force foload step index with varying mesh resolution: (c) lower limit, (d) upper limit 
Upper and lower ECR boundaries with different meshing resolutions are plotted in Fig. 9 (c) and 9 (d) respectively. See the lower boundary first, it is not continued, this is logical considering the FE model has limit elements in contact. Some abrupt jumps are found, which may come from:

- changes in the number of spots, new spots can appear and some spots can merge;

- electrical transport regime changing caused by the dimension evolvement of the spots.

Conversely, upper limit curves are smooth. As explained above, Au-Au contact have the asperities experience highly plastic deformation, so that pressure defined as $p=F / A$ is almost constant (see Fig. 5(b)); therefore contact area evolves smoothly, and so does the upper limit of ECR.

It is expected that the upper limit is quite close to the real constriction resistance here. Indeed, the contact zone is located in a small region, as opposed to the conforming contact problem; which causes high electrical interaction.

Again, F1 and F2 simulations lead to the close ECR values. This indicates that using a finer mesh would likely not provide much more accuracy.

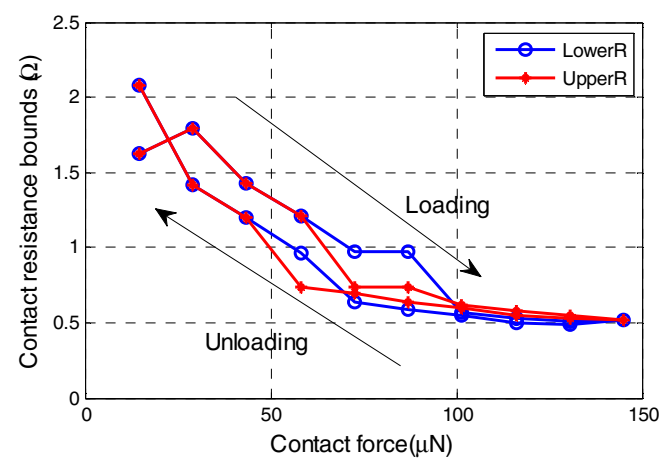

(a)

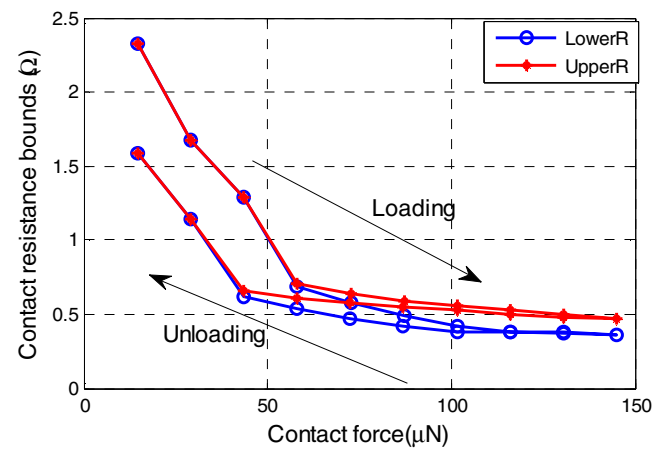

(b)

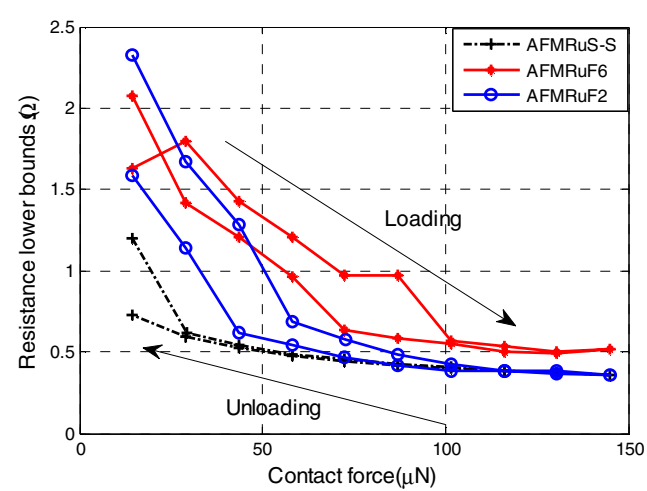

(c)

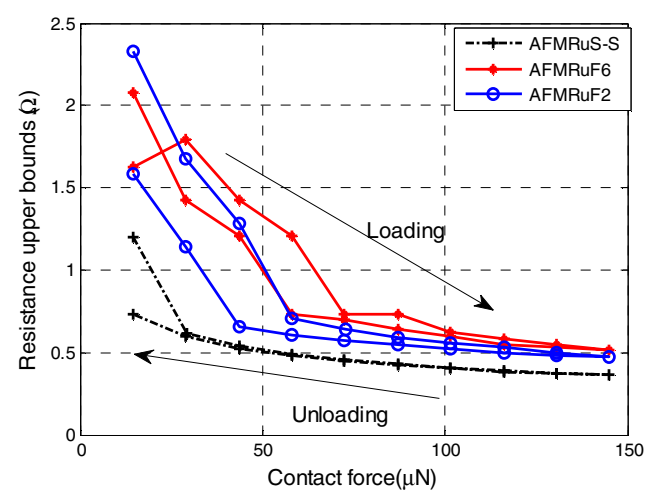

(d)

Figure 10. Contact resistance for Ru-Ru contact: ECR limits vs. force for the models with the (a) coarsest mesh and (b) finest mesh; ECR boundary with varying mesh resolution: (c) lower limit, (d) upper limit

The results for Ru-Ru contact are presented in Fig. 10. Like $\mathrm{Au}-\mathrm{Au}$ contact, the ECR limits get looser with finer meshing and at lower force. While Ru-Ru contact exhibit a very similar behavior during loading and unloading, ECR deviation is appreciable, especially at low force.

From Fig. 9 and Fig. 10, it is expected that the mesh grid size has more impact on ECR predicting at low force, while little influence at the work force of microswitchs.

\section{B. Evolution of Contact Spots}

As illustrated in part IV, contact resistance is slightly affected by the mesh grid of simulations. However, Fig. 11 shows that the number of contact spots is directly affected by mesh resolution. This was expected and it is likely that the finest mesh used here is not sufficient to have an accurate result.

The number of contact spots and the radius of each spot for contact $\mathrm{Au}$ and $\mathrm{Ru}$ are summed up in Fig. 12; the plots are based on the simulations with the finest mesh. Comparing the two graphs first shows the effects of hardness: Ru contact yields fewer contact spots and smaller radii, under the same load. 


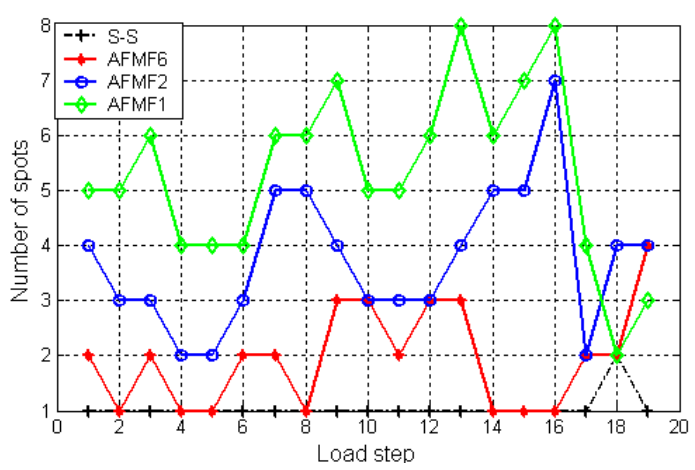

(a)

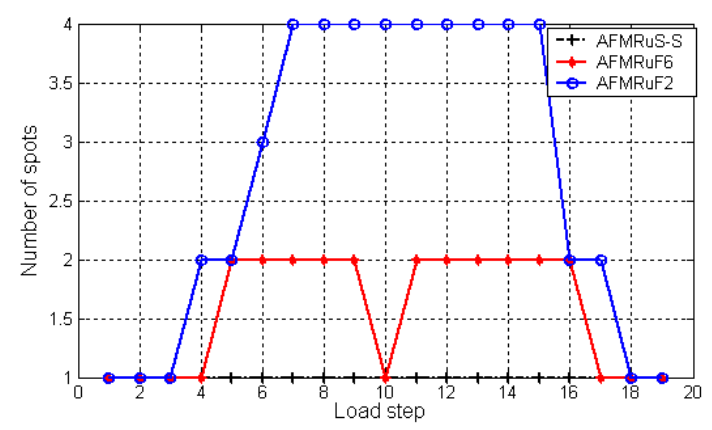

(b)

Figure 11. Number of spots in contact predicted by different FE models, with varying mesh resolution for material: (a) $\mathrm{Au}$, (b) $\mathrm{Ru}$

$\mathrm{Au}-\mathrm{Au}$ contact simulation shows a simple global evolution: several contact spots appear in the first load step, then further loading leads to merging spots while new spots appear, keeping the total number of spots roughly constant. The same behavior is assumed for $\mathrm{Ru}-\mathrm{Ru}$ contact, although it takes 7 out of 10 load steps to stabilize the number of spots. For both materials, the contact resistance is eventually dominated by only one spot, from load step 5 for $\mathrm{Au}, 7$ for $\mathrm{Ru}$.

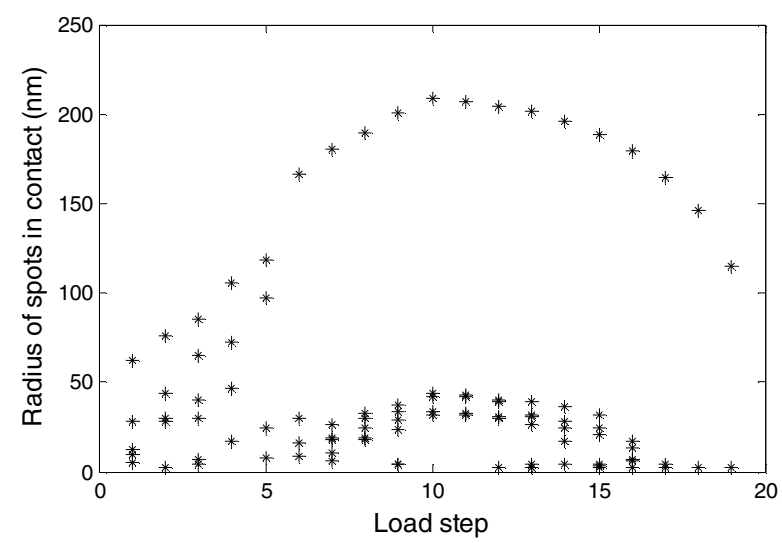

(a)

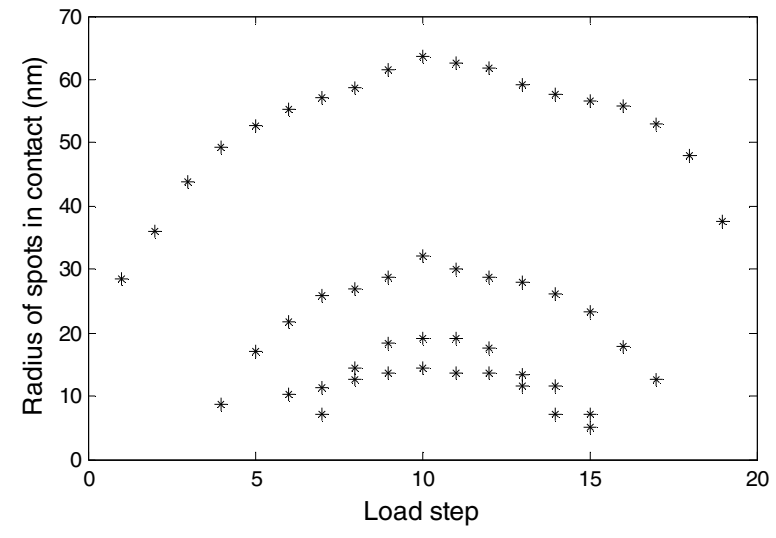

(b)

Figure 12. Development of the spots in contact for material (a) $\mathrm{Au}$, (b) $\mathrm{Ru}$

\section{CONCLUSIONS}

A determinist FE model is used to predict the frictionless contact behavior and the contact resistance for ohmic microswitches in a loading-unloading cycle. The model assumes elastic-plastic deformation, and the effect of strain hardening is taken into account. The influence of roughness is studied by varying mesh resolution and asperity height. The results show that the displacement and the contact area are sensitive to the roughness, but ECR deviation remains quite limited. Compared to gold, the harder material ruthenium has less plasticity deformation and hysteresis.

The evolution of contact spots has been shown for the materials $\mathrm{Au}$ and $\mathrm{Ru}$. The number of spots becomes roughly constant after some load steps, and the contact resistance is eventually dominated by only one spot. Harder material leads to less spots in contact and smaller spot radius. Computing accurately the number of contact spots requires very fine mesh grids; the finest models lead to outrageous solving duration.

In hope of predicting contact temperature and heat distribution, modeling the thermo-electrical behavior of the contact region is likely to require roughness to a much greater extent.

\section{REFERENCES}

[1] J. A. Greenwood and J. B. P Willamson, "Contact of nominally flat surfaces," Proc. Roy. Soc (London), series A, vol. 295, pp. 300-319, 1966.

[2] L. Kogut and I. Etsion, "A finite element based elastic-plastic model for the contact of rough surfaces," Tribology Transactions, vol. 46, pp. 383390, 2003.

[3] Y. Zhao and L. Chang, "A model of asperity interactions in elasticplastic contact of rough surfaces," Journal of Tribology, vol. 123, pp.857-864, 2001.

[4] R. L. Jackson and I. Green "A statistical model of elasto-plastic asperity contact between rough surfaces," Tribology International, vol. 39, pp. 906-914, 2006.

[5] A. Majumdar and B. Bhushan, "Fractal model of elastic-plastic contact between rough surfaces," Journal of Tribology, vol. 113, pp. 1-11, 1991.

[6] P. Sahoo and N. Ghosh, "Finite element contact analysis of fractal surfaces," Journal of Physics D: Applied Physics, vol. 40, pp. 42454252, 2007. 
[7] O. Rezvanian, M. A. Zikry, C. Brown, and J. Krim, "Surface roughness, asperity contact, and gold RF MEMS switch behavior," Journal of Micromechanics and Microengineering, vol. 17, pp. 2006-2015, 2007.

[8] R. L. Jackson and J. L. Streator, "A multi-scale model for contact between rough surfaces," Wear, vol. 261, pp. 1337-1347, 2006.

[9] W. E. Wilson, S. V. Angadi, and R. L. Jackson, "Surface separation and contact resistance considering sinusoidal elastic-plastic multi-scale rough surface contact, " Wear, vol. 268, pp. 190-201, 2010.

[10] F. Pennec D. Peyrou, et al, "DC contact modeling of electrostatically actuated switches with low voltage, ", 20th MicroMechanics Europe Workshop (MME2009), 2009.

[11] V. Brizmer, Y. Zait, Y. Kligerman, and I. Etsion "The effect of contact conditions and material propertis on elastic-plastic spherical contact," Journal of Mechanics of Materials and Structures, vol. 1, pp. 865-879, 2006.

[12] A. Broue, T. Fourcade, et al, "Validation of bending tests by nanoindentation for micro-contact analysis of MEMS switches," Journal of Micromechanics and Microengineering, vol. 20, pp. 1-8, 2010.

[13] L. Li, I. Etsion, and F. E. Talke, "Elastic-plastic spherical contact modeling including roughness effects," Tribology Letters, vol. 40, pp. 357-363, 2010.

[14] J. A. Greenwood and J. H. Tripp, "The elastic contact of rough spheres," J. Appl. Mech., vol. 34, pp. 153-159, 1967.

[15] F. S. Wang, J. M. Block, et al, "A multilevel model for elastic-plastic contact between a sphere and a flat rough surface, ” J. Tribol. Trans. ASME, vol. 131, p. 021409-1, 2009.

[16] M. Bahrami, M. M. Yovanovich, and J. R. Culham, "A compact model for spherical rough contacts, ", Proceedings of International Joint Tribology Conference 2004, ASME/STLE, 2004.

[17] D. Cohen, Y. Kligerman, and I. Etsion, "The effect of surface roughness on static friction and junction growth of an elastic-plastic spherical contact, ", J. Tribol. Trans. ASME, vol. 131, p. 021404, 2009.

[18] R. L. Jackson and L. Kogut, "A comparison of flattening and indentation approaches for contact mechanics modeling of single asperity contacts," Journal of Tribology, vol. 128, pp. 209-212, 2006.

[19] I. Etsion, Y. Kligerman, and Y. Kadin., "Unloading of an elastic-plastic loaded spherical contact," International Journal of Solids and Structures, vol. 42, pp. 3716-3729, 2005.
[20] A. Ovcharenko, G. Halperin, G. Verberne, and I. Etsion, "In situ investigation of the contact area in elastic-plastic spherical contact during loading-unloading," Tribology Letters, vol. 25, pp. 153-160, 2006.

[21] Y. Du, L. Chen, N. E. McGruer, G. G. Adams, and I. Etsion, "A finite element model of loading and unloading of an asperity contact with adhesion and plasticity," Journal of Colloid and Interface Science, vol. 312, pp. 522-528, 2007.

[22] J. H. Zhao, S. Nagao, and Z. L. Zhang, "Loading and unloading of a spherical contact: From elastic to elastic-perfectly plastic materials," International Journal of Mechanical Sciences, vol. 56, pp. 70-76, 2012.

[23] B. Chatterjee and P. Sahoo, "Effect of strain hardening on unloading of a deformable sphere loaded against a rigid flat - a finite element study," International Journal of Engineering and Technology, vol. 2(4), pp. 225233, 2010.

[24] Y. Kadin, Y. Kligerman, and I. Etsion, "Unloading an elastic-plastic contact of rough surfaces," Journal of Mechanics and Physics of Solid, vol. 54, pp. 2652-2674, 2006.

[25] L. Kogut and K. Komvopoulos, "Electrical contact resistance theory for conductive rough surfaces," Journal of Applied Physics, vol. 94, pp. 3153-3162, 2003.

[26] R. L. Jackson and I. Green, "A Finite element study of elasto-plastic hemispherical contact against a rigid flat," Journal of Tribology, vol. 127, pp. 343-354, 2005.

[27] R. S. Timsit, "Electrical conduction through small contact spots," Transactions on Components and Packaging Technologies, vol. 29, pp. 727-734, 2006.

[28] B. Chatterjee and P. Sahoo, "Effect of strain hardening on elastic-plastic contact of a deformable sphere against a rigid flat under full stick contact condition," Advances in Tribology, vol. 2012, pp. 1-8, 2012.

[29] L. Boyer, S. Nöel, and F. Houzé., "Constriction resistance of a multispot contact: An improved analytical expression," 36th IEEE Holm Conf. and the 15th International Conference on Electrical Contacts, 1990.

[30] S. Majumder, N.E. McGruer, G. G.Adams, P. M. Zavracky, R. H. Morrison, and J. Krim, "Study of contacts in an electrostatically actuated microswitch," Sensors and Actuators A, vol. 93, pp. 19-26, 2001.

[31] L. Kogut and I. Etsion, "Elastic-plastic contact analysis of a sphere and a rigid flat,"Journal of Applied Mechanics, vol. 69, pp. 657-662, 2002. 